\title{
Static LQG Teams with Countably Infinite Players
}

\author{
Aditya Mahajan, Nuno C. Martins, and Serdar Yüksel
}

\begin{abstract}
In static LQG teams with finite number of players, there is no loss of optimality in restricting attention to affine control strategies. This result, in turn, implies that affine control strategies are globally optimal for finite horizon partially nested LQG teams. The standard proof of optimality of affine strategies does not generalize to the setting where the team has countably infinite players and the cost function is the average expected cost per player. Consequently, it is not clear whether affine control strategies are globally optimal for infinite horizon partially nested LQG teams. We identify sufficient conditions under which affine control strategies are globally optimal for static teams with countably infinite players. An example is included.
\end{abstract}

\section{INTRODUCTION}

Decentralized decision making is an increasingly relevant area of research with applications ranging from networked control systems, sensor networks, traffic management, vehicle coordination, to sociology and interaction of masses, as well as economics.

Consider a collection of players where each player has access to a local information variable and they collective wish to minimize a common cost function. Assume that the players agree on the system (that is, the probability space on which the system is defined, and the strategy and action spaces). Such a collection is called a team; when one of these conditions is not satisfied, the collection is said to engage in a game. A team is dynamic if the information of one player is affected by the decision rule of some other player. A team is sequential if the order in which the players act can be specified before the system starts running. Witsenhausen [1] provided the following characterization of information structures in a dynamic sequential team: Under a centralized information structure, all players have the same information. Under a quasi-classical information structure whenever the information of any player, say player $j$, depends on the decision rule of another player, say player $k$, then player $j$ has access to the information available to player $k$. An information structure that is neither classical nor quasiclassical is called non-classical.

Team decision theory has its roots in both control theory and economics. Marschak [2] was perhaps the first to introduce the basic elements of (static) teams, and to provide the first steps toward the development of a team theory. Radner [3] followed with a mathematical formulation and developed

A. Mahajan is with the Dept. of ECE, McGill University, Canada. (aditya.mahajan@mcgill.ca).

N. C. Martins is with the Dept. of ECE and the ISR, University of Maryland, College Park. (nmartinseisr.umd.edu).

S. Yüksel is with the Dept. of Mathematics and Statistics, Queen's University at Kingston, Canada. (yukselemast.queensu.ca). results to some classes of static teams, establishing connections between person-by-person optimality, stationarity, and team-optimality (concepts that we will visit in further detail). Marschak's and Radner's collaborative work culminated in [4]. Contributions of Witsenhausen [1], [5]-[8] on dynamic teams and characterization of information structures have been crucial in the progress of our understanding of dynamic teams. For a detailed review of the literature and classification of information structures we refer the reader to [9].

For static linear quadratic team (LQG) problems, [3] and [10] established the optimality of person-by-personoptimal policies. Ho and Chu [11] made the observation that this result holds for partially nested LQG teams: partially observed LQG teams can be reduced to static teams by a transformation of the cost. For further extensions see [6].

Along a separate line of thought, when one deals with decentralization, information has been interpreted by nonstochastic sparsity conditions. In such formulations, a player has access to only a subset of variables in the system. The goal is to minimize an operator norm in an infinite horizon problem when the controllers have been restricted to be linear. In a recent tutorial paper [12], we highlighted some of the differences between these two approaches. Relevant to our paper are the recent results on frequency domain characterization of solutions to deterministic teams, as well as quadratic stochastic dynamic teams under the a priori restriction to linear policies [13], [14], [15], [16], [17].

However, there has been no conclusive research on the interaction between the stochastic control approach (described above) and the operator theoretic sparsity-based approach. One reason is that the tools, techniques and the definitions have been very different, as has been highlighted in [12]. In general, these two approaches impose different modelling assumptions. In the stochastic control approach, a probabilistic model is assumed for the noise, no restriction is imposed on the dynamics, and the objective is to minimize the expected total or average cost. In the operator theoretic sparsity-based approach, no restriction is imposed on the noise model, the dynamics are assumed to be linear, and the objective is to minimize some norm on the cost process. The stochastic control approach does not impose any restriction on the form of the controller, while the sparsity-constrained approach restricts attention to linear controllers.

Given these differences in the modeling assumptions and the solution concepts, a natural question is whether one can compare the results of the two approaches at all. In this paper, we present preliminary results to address this question.

The closest point of contact between the two approaches is the LQG systems with partially nested information struc- 
ture studied using a stochastic control approach and the quadratically invariant systems with $\mathcal{H}_{2}$ norm studied using sparsity-constrained approach. It is not hard to see that the LQG optimal control problem can be posed as a $\mathcal{H}_{2}$-control problem and vice versa. To make a precise comparison, we need to pose the same problem using the two approaches.

As mentioned above, Ho and Chu [11] studied finite horizon LQG systems with partially nested information structures and showed that there is no loss of optimality in restricting attention to affine controllers. The critical steps of the proof were: (i) we can transform the finite horizon dynamical system into a static team with finite players using a linear (information) transformation; and (ii) affine strategies are globally optimal for static teams with finite players [3].

This approach does not directly extend to infinite horizon partially nested teams. We can still use a linear transformation to convert partially nested team into a static team, but the resulting static team will have countably infinite players. Radner's result for static teams is only valid for teams with finite players.

In this paper, we generalize the theory due to Radner [3] and Krainak, Speyer, and Marcus [10] to teams with countably infinitely many players. We establish sufficient conditions on the global optimality of affine policies. We then present an illustrative example.

\section{STATIC LQG TEAMS}

In this section, we formulate an infinite player static LQG team as a limit of finite player LQG teams. We use the following notation: subscripts denote the index of the player and superscripts denote the number of players in the team; e.g., $y_{i}^{n}$ denotes the observation of player $i$ in a team with $n$ players. Bold face letters denote vectors: $\boldsymbol{u}^{n}$ denotes the vector $\left(u_{1}^{n}, \ldots, u_{n}^{n}\right) ; \boldsymbol{u}^{\infty}$ denotes the infinitedimensional vector $\left(u_{1}^{\infty}, u_{2}^{\infty}, \ldots\right)$. Similarly, $\boldsymbol{w}^{n}$ denotes the vector $\left(w_{0}^{n}, w_{1}^{n}, \ldots, w_{n}^{n}\right)$ and $\boldsymbol{w}^{\infty}$ denotes the vector $\left(w_{0}^{\infty}, w_{1}^{\infty}, \ldots\right)$. For the infinite dimensional vector $\boldsymbol{u}^{\infty}$, the notation $\left.\boldsymbol{u}^{\infty}\right|_{n}$ denotes the $n$-dimensional projection $\left(u_{1}^{\infty}, \ldots, u_{n}^{\infty}\right)$. Similarly, for the infinite dimensional vector $\boldsymbol{w}^{\infty}$, the notation $\left.\boldsymbol{w}^{\infty}\right|_{n}$ denotes the $(n+1)$-dimensional projection $\left(w_{0}^{\infty}, w_{1}^{\infty}, \ldots, w_{n}^{\infty}\right)$.

\section{A. Team with finite players}

In an $n$-player static LQG team, player $i$ observes

$$
y_{i}^{n}=A_{i}^{n} w_{0}^{n}+D_{i}^{n} w_{i}^{n}
$$

and chooses a control action

$$
u_{i}^{n}=\gamma_{i}^{n}\left(y_{i}^{n}\right)
$$

The variables $\left(w_{i}^{n}, y_{i}^{n}\right.$, and $u_{i}^{n}$ are vectors of dimensions $d_{w}$, $d_{y}$, and $d_{u}$, respectively. ${ }^{1}$ The variables $\left(w_{0}^{n}, w_{1}^{n}, \ldots, w_{n}^{n}\right)$ are zero-mean jointly Gaussian random variables with covariance $\Sigma_{i j}=\mathbb{E}\left[w_{i}^{n}\left(w_{j}^{n}\right)^{\top}\right]$ that does not depend on $n$.

\footnotetext{
${ }^{1}$ We assume that the dimension does not depend on $i$ for simplicity of exposition. The critical assumption here is that the dimension does not depend on $n$.
}

The performance of a team strategy $\gamma^{n}=\left(\gamma_{1}^{n}, \ldots, \gamma_{n}^{n}\right)$ is quantified by an expected quadratic cost

$$
J^{n}\left(\boldsymbol{\gamma}^{n}\right)=\frac{1}{n} \mathbb{E}\left[c^{n}\left(\boldsymbol{w}^{n}, \boldsymbol{u}^{n}\right)\right]
$$

where $\boldsymbol{w}^{n}=\left(w_{0}^{n}, w_{1}^{n}, \ldots, w_{n}^{n}\right), \boldsymbol{u}^{n}=\left(u_{1}^{n}, \ldots, u_{n}^{n}\right)$, and

$$
\begin{aligned}
c^{n}\left(\boldsymbol{w}^{n},\right. & \left.\boldsymbol{u}^{n}\right) \\
& =\sum_{i=1}^{n} \sum_{j=1}^{n}\left(u_{i}^{n}\right)^{\top} Q_{i j}^{n} u_{j}^{n}+\sum_{i=1}^{n} \sum_{k=0}^{n}\left(u_{i}^{n}\right)^{\top} P_{i k}^{n} w_{k}^{n}
\end{aligned}
$$

where $Q_{i j}^{n}$ and $P_{i k}^{n}$ are matrices of appropriate dimensions and $\boldsymbol{Q}^{n}=\left[Q_{i j}\right]$ is positive symmetric.

In contrast to the formulation in [3], we normalize the cost (3) by $1 / n$. Such a normalization is needed to extend the formulation to infinite number of players. As a consequence of this normalization, additional technical conditions are required to extend the result of system with finite players to the system with infinite players. We present such conditions in this paper.

With a slight abuse of notation, we also use $c^{n}$ to denote the following:

$$
\begin{aligned}
& c^{n}\left(\boldsymbol{w}^{n}, \boldsymbol{\gamma}^{n}\right)=\left.c^{n}\left(\boldsymbol{w}^{n}, \boldsymbol{u}^{n}\right)\right|_{u_{i}^{n}=\gamma_{i}^{n}\left(A_{i}^{n} w_{0}^{n}+D_{i}^{n} w_{i}^{n}\right), i \in\{1, \ldots, n\}} \\
& \begin{array}{r}
c^{n}\left(\boldsymbol{w}^{n}, \boldsymbol{\gamma}_{-i}^{n}, u_{i}^{n}\right) \\
\quad=\left.c^{n}\left(\boldsymbol{w}^{n}, \boldsymbol{u}^{n}\right)\right|_{u_{j}^{n}=\gamma_{j}^{n}\left(A_{j}^{n} w_{0}^{n}+D_{j}^{n} w_{j}^{n}\right), j \in\{1, \ldots, n\} \backslash\{i\}}
\end{array}
\end{aligned}
$$

Definition 1 (Globally optimal strategy) A strategy $\gamma^{n}$ is globally optimal if for all possible choices $\tilde{\gamma}^{n}$ of the strategy for all players

$$
J^{n}\left(\gamma^{n}\right) \leq J^{n}\left(\tilde{\gamma}^{n}\right)
$$

Definition 2 (Person-by-person optimal strategy) A strategy $\gamma^{n}$ is person-by-person optimal if for all players $i$ and all possible choices $\tilde{\gamma}_{i}^{n}$ of the strategy for player $i$

$$
J^{n}\left(\gamma^{n}\right) \leq J^{n}\left(\tilde{\gamma}_{i}^{n}, \gamma_{-i}^{n}\right)
$$

\section{B. Optimality conditions for teams with finite players}

Consider two arbitrary policies $\gamma^{n}$ and $\tilde{\gamma}^{n}$. For a particular realization of $\boldsymbol{w}^{n}$, let $u_{i}^{n}=\gamma_{i}^{n}\left(y_{i}^{n}\right)$ and $\tilde{u}_{i}^{n}=\tilde{\gamma}_{i}^{n}\left(y_{i}^{n}\right)$ and define $h_{i}^{n}=\tilde{u}_{i}^{n}-u_{i}^{n}$. Then, following the arguments of [10], we get that

$$
\begin{aligned}
& J^{n}\left(\tilde{\boldsymbol{\gamma}}^{n}\right)-J^{n}\left(\boldsymbol{\gamma}^{n}\right)=\frac{1}{n} \mathbb{E}\left[c^{n}\left(\boldsymbol{w}^{n}, \tilde{\boldsymbol{u}}^{n}\right)\right]-\frac{1}{n} \mathbb{E}\left[c^{n}\left(\boldsymbol{w}^{n}, \boldsymbol{u}^{n}\right)\right] \\
& \quad=\frac{1}{n} \mathbb{E}\left[c^{n}\left(\boldsymbol{w}^{n}, \boldsymbol{u}^{n}+\boldsymbol{h}^{n}\right)-c^{n}\left(\boldsymbol{w}^{n}, \boldsymbol{u}^{n}\right)\right] \\
& \quad \geq \lim _{\alpha \rightarrow 0} \frac{1}{n} \mathbb{E}\left[\frac{1}{\alpha}\left[c^{n}\left(\boldsymbol{w}^{n}, \boldsymbol{u}^{n}+\alpha \boldsymbol{h}^{n}\right)-c^{n}\left(\boldsymbol{w}^{n}, \boldsymbol{u}^{n}\right)\right]\right] \\
& \quad=\frac{1}{n} \mathbb{E}\left[\sum_{i=1}^{n}\left(h_{i}^{n}\right)^{\boldsymbol{\top}}\left[2 \sum_{j=0}^{n} Q_{i j}^{n} u_{j}^{n}+\sum_{k=0}^{n} P_{i k}^{n} w_{k}^{n}\right]\right] \\
& \quad=: \Delta^{n}\left(\boldsymbol{\gamma}^{n}, \tilde{\boldsymbol{\gamma}}^{n}\right)
\end{aligned}
$$

where (6) follows because $c^{n}$ is convex in $\boldsymbol{u}^{n}$ and a version of the monotone convergence theorem applies (see [10]) and (7) follows by substituting (4) in (6). Furthermore,

$J^{n}\left(\tilde{\gamma}_{i}, \gamma_{-i}^{n}\right)-J^{n}\left(\gamma^{n}\right)$ 


$$
\begin{aligned}
& =\frac{1}{n} \mathbb{E}\left[c^{n}\left(\boldsymbol{w}^{n}, \tilde{u}_{i}^{n}, \boldsymbol{u}_{-i}^{n}\right)\right]-\frac{1}{n} \mathbb{E}\left[c^{n}\left(\boldsymbol{w}^{n}, \boldsymbol{u}^{n}\right)\right] \\
& =\frac{1}{n} \mathbb{E}\left[c^{n}\left(\boldsymbol{w}^{n}, u_{i}^{n}+h_{i}^{n}, \boldsymbol{u}_{-i}^{n}\right)-c^{n}\left(\boldsymbol{w}^{n}, \boldsymbol{u}^{n}\right)\right] \\
& \geq \lim _{\alpha \rightarrow 0} \frac{1}{n} \mathbb{E}\left[\frac{1}{\alpha}\left[c^{n}\left(\boldsymbol{w}^{n}, u_{i}^{n}+\alpha h_{i}^{n}, \boldsymbol{u}_{-i}^{n}\right)-c^{n}\left(\boldsymbol{w}^{n}, \boldsymbol{u}^{n}\right)\right]\right] \\
& =\frac{1}{n} \mathbb{E}\left[\left(h_{i}^{n}\right)^{\boldsymbol{\top}}\left[2 \sum_{j=0}^{n} Q_{i j}^{n} u_{j}^{n}+\sum_{k=0}^{n} P_{i k}^{n} w_{k}^{n}\right]\right] \\
& =: \Delta_{i}^{n}\left(\boldsymbol{\gamma}^{n}, \tilde{\boldsymbol{\gamma}}^{n}\right)
\end{aligned}
$$

where (10) follows because $c^{n}$ is a convex in $u_{i}^{n}$ and (11) follows by substituting (4) in (10).

Remark 1 A strategy $\gamma^{n}$ is globally optimal if for any other strategy $\tilde{\gamma}^{n}, J^{n}\left(\tilde{\gamma}^{n}\right)-J^{n}\left(\gamma^{n}\right) \geq 0$. Thus, a sufficient condition for global optimality of $\gamma$ is that $\Delta^{n}\left(\gamma^{n}, \tilde{\gamma}^{n}\right) \geq 0$ holds for any strategy $\tilde{\gamma}^{n}$.

Remark 2 A strategy $\gamma^{n}$ is person-by-person optimal if for any other strategy $J^{n}\left(\tilde{\gamma}_{i}, \gamma_{-i}^{n}\right)-J^{n}\left(\gamma^{n}\right) \geq 0$ for all $i$. Thus, a sufficient condition for person-by-person optimality of $\gamma$ is that $\Delta_{i}^{n}\left(\gamma^{n}, \tilde{\gamma}^{n}\right) \geq 0$ holds for any strategy $\tilde{\gamma}^{n}$ and for all $i$.

Remark 3 If $\Delta_{i}^{n}\left(\gamma^{n}, \tilde{\gamma}^{n}\right) \geq 0$ for all $i$, then $\Delta^{n}\left(\gamma^{n}, \tilde{\gamma}^{n}\right) \geq$ 0 . Thus, if a strategy satisfies the sufficient condition of Remark 2, then it is also globally optimal.

Remark 4 A sufficient condition for $\Delta_{i}^{n}\left(\boldsymbol{\gamma}^{n}, \tilde{\gamma}^{n}\right) \geq 0$ for all $i$ is that

$$
\mathbb{E}\left[2 \sum_{j=0}^{n} Q_{i j}^{n} u_{j}^{n}+\sum_{k=0}^{n} P_{i k}^{n} w_{k}^{n} \mid y_{i}^{n}\right]=0, \quad i=1, \ldots, n .
$$

The discussion so far has not used the fact that the primitive random variables are Gaussian. The next result relies on pritive variables being Gaussian.

Proposition 1 (Radner) Let $R_{i j}^{n}=\mathbb{E}\left[y_{i}^{n}\left(y_{i}^{n}\right)^{\top}\right]$ and $S_{k i}^{n}=$ $\mathbb{E}\left[w_{k}^{n}\left(y_{i}^{n}\right)^{\top}\right]$. The system of equations (13) has a solution of the form $^{2}$

$$
u_{i}^{n}=\gamma_{i}^{n}\left(y_{i}^{n}\right)=H_{i}^{n} y_{i}^{n}
$$

where $H_{i}^{n} \in \mathbb{R}^{d_{u} \times d_{y}}$ is given by the solution of the following system of linear (matrix) equations

$$
2 \sum_{j=1}^{n} Q_{i j}^{n} H_{i}^{n} R_{j i}^{n}+\sum_{k=0}^{n} P_{i k}^{n} S_{k i}^{n}=0, \quad i=1, \ldots, n .
$$

Remark 4 implies that the control law (14) is person-byperson optimal. Remark 3 implies that it is globally optimal.

The system of equations (15) may be written in a compact form using vectorization. For a matrix $A$, let $\operatorname{vec}(A)$ denote the column vector formed by stacking the columns of $A$. Then, similar to the discussion in [18, Eq. (8)], (15) may be simplified as follows:

\footnotetext{
${ }^{2}$ We assumed that the primitive random variable is zero mean, so our solution does not have any affine term. In general, if $w_{0}^{n}$ is not zero mean, (14) will have an affine correction term. See [3], or [9] for details.
}

Corollary 1 The solution of the form (14) for the system of equations (13) is given by

$$
2 \Gamma^{n} \vec{H}^{n}+\eta^{n}=0
$$

where $\Gamma^{n}=\left[\Gamma_{i j}^{n}\right], \Gamma_{i j}^{n}=R_{i j}^{n} \otimes Q_{i j}^{n}$ and $\otimes$ denotes the Kronecker product, $\vec{H}^{n}=\operatorname{vec}\left[H_{1}^{n}|\cdots| H_{n}^{n}\right]$, and

$$
\eta^{n}=\operatorname{vec}\left[\sum_{k=0}^{n} P_{0 k}^{n} S_{k o}^{n}|\cdots| \sum_{k=0}^{n} P_{n k}^{n} S_{k n}^{n}\right] .
$$

Substituting (14) in (3) and using the above vector notation gives that

$$
\begin{aligned}
J^{n}\left(\gamma^{n}\right) & =\frac{1}{n}\left[\left(\vec{H}^{n}\right)^{\top} \Gamma^{n} \vec{H}^{n}+\left(\vec{H}^{n}\right)^{\top} \eta^{n}\right] \\
& =-\frac{1}{4 n}\left(\eta^{n}\right)^{\top}\left(\Gamma^{n}\right)^{-1} \eta^{n} .
\end{aligned}
$$

PROOF Vectorizing (15) gives that for $i=1, \ldots, n$,

$$
2 \sum_{j=1}^{n} \operatorname{vec}\left(Q_{i j}^{n} H_{i}^{n} R_{j i}^{n}\right)+\operatorname{vec}\left(\sum_{k=0}^{n} P_{i k}^{n} S_{k i}^{n}\right)=0 .
$$

Using the fact that for any matrices $A, B$, and $C$ of appropriate sizes, $\operatorname{vec}(A B C)=\operatorname{vec}\left(C^{\top} \otimes A\right) \operatorname{vec}(B)$ and the definitions of $\vec{H}^{n}, \Gamma^{n}$ and $\eta^{n}$, we get (16).

Similarly, vectorizing (4) gives

$$
\begin{array}{r}
\mathbb{E}\left[c^{n}\left(\boldsymbol{w}^{n}, \boldsymbol{\gamma}^{n}\right)\right]=\sum_{i=1}^{n} \operatorname{vec}\left(H_{i}^{n}\right)\left[\sum_{j=1}^{n} \operatorname{vec}\left(Q_{i j}^{n} H_{j}^{n} R_{j i}^{n}\right)\right. \\
\left.+\operatorname{vec}\left(\sum_{k=0}^{n} P_{i k}^{n} S_{k i}^{n}\right)\right] .
\end{array}
$$

Using the above property of $\operatorname{vec}(A B C)$, we get (18)

\section{Team with infinite players}

In an infinite player static LQG team, we assume that the primitive random variables $\boldsymbol{w}^{\infty}=\left(w_{0}^{\infty}, w_{1}^{\infty}, \ldots\right)$ are independent Gaussian random variables. The observation and control action of player $i$ are given by (1) and (2). The performance of a team strategy $\gamma^{\infty}=\left(\gamma_{1}^{\infty}, \gamma_{2}^{\infty}, \ldots\right)$ is quantified by

$$
J^{\infty}\left(c^{\infty}\right)=\limsup _{n \rightarrow \infty} \frac{1}{n} \mathbb{E}\left[c^{n}\left(\left.\boldsymbol{w}^{\infty}\right|_{n},\left.\boldsymbol{u}^{\infty}\right|_{n}\right)\right]
$$

where $c^{n}$ is given by (4).

We are interested in deriving conditions for optimality for such teams. The analysis of Section II-B does not directly apply. We illustrate this point in the next section and identify a different sufficient condition for optimality.

D. Optimality conditions for teams with countably infinite players

Consider two arbitrary policies $\gamma^{\infty}$ and $\tilde{\gamma}^{\infty}$. For a particular realization of $\boldsymbol{w}^{\infty}$, let $u_{i}^{\infty}=\gamma_{i}^{\infty}\left(y_{i}^{\infty}\right)$ and $\tilde{u}_{i}^{\infty}=$ $\tilde{\gamma}_{i}^{\infty}\left(y_{i}^{\infty}\right)$ and define $h_{i}^{\infty}=\tilde{u}_{i}^{\infty}-u^{\infty}$. Let $n_{m}$ be a subsequence such that

$$
J^{\infty}\left(\gamma^{\infty}\right)=\limsup _{n \rightarrow \infty} \frac{1}{n} \mathbb{E}\left[c^{n}\left(\left.\boldsymbol{w}^{\infty}\right|_{n},\left.\boldsymbol{u}^{\infty}\right|_{n}\right)\right]
$$




$$
=\lim _{m \rightarrow \infty} \frac{1}{n_{m}} \mathbb{E}\left[c^{n_{m}}\left(\left.\boldsymbol{w}^{\infty}\right|_{n_{m}},\left.\boldsymbol{u}^{\infty}\right|_{n_{m}}\right)\right]
$$

Then,

$$
\left.\begin{array}{l}
J^{\infty}\left(\tilde{\boldsymbol{\gamma}}^{\infty}\right)-J^{\infty}\left(\boldsymbol{\gamma}^{\infty}\right) \\
=\limsup _{n \rightarrow \infty} \frac{1}{n} \mathbb{E}\left[c^{n}\left(\left.\boldsymbol{w}^{\infty}\right|_{n},\left.\tilde{\boldsymbol{u}}^{\infty}\right|_{n}\right)\right] \\
\quad-\limsup _{n \rightarrow \infty} \frac{1}{n} \mathbb{E}\left[c^{n}\left(\left.\boldsymbol{w}^{\infty}\right|_{n},\left.\boldsymbol{u}^{\infty}\right|_{n}\right)\right] \\
\geq \limsup _{m \rightarrow \infty} \frac{1}{n_{m}} \mathbb{E}\left[c^{n_{m}}\left(\left.\boldsymbol{w}^{\infty}\right|_{n_{m}},\left.\tilde{\boldsymbol{u}}^{\infty}\right|_{n_{m}}\right)\right] \\
\quad-\lim _{m \rightarrow \infty} \frac{1}{n_{m}} \mathbb{E}\left[c^{n_{m}}\left(\left.\boldsymbol{w}^{\infty}\right|_{n_{m}},\left.\boldsymbol{u}^{\infty}\right|_{n_{m}}\right)\right] \\
=\limsup _{m \rightarrow \infty} \frac{1}{n_{m}} \mathbb{E}\left[c^{n_{m}}\left(\left.\boldsymbol{w}^{\infty}\right|_{n_{m}},\left.\tilde{\boldsymbol{u}}^{\infty}\right|_{n_{m}}\right)\right. \\
\geq \limsup _{m \rightarrow \infty} \operatorname{limm}_{\alpha \rightarrow 0} \frac{1}{n_{m}}\left[\frac{1}{\alpha}\left[c^{n_{m}}\left(\left.\boldsymbol{w}^{\infty}\right|_{n_{m}},\left.\boldsymbol{u}^{\infty}\right|_{n_{m}}\right)\right]\right. \\
\left.\qquad\left.\boldsymbol{w}^{\infty}\right|_{n_{m}},\left.\boldsymbol{u}^{\infty}\right|_{n_{m}}+\left.\boldsymbol{h}^{\infty}\right|_{n_{m}}\right) \\
=\limsup _{m \rightarrow \infty} \Delta^{n_{m}}\left(\left.\boldsymbol{\gamma}^{\infty}\right|_{n_{m}},\left.\tilde{\boldsymbol{\gamma}}^{\infty}\right|_{n_{m}}\right) \\
=\limsup _{m \rightarrow \infty} \frac{1}{n_{m}} \mathbb{E}\left[\sum_{i=1}^{n_{m}}\left(\left.\boldsymbol{w}^{\infty}\right|_{n_{m}},\left.\boldsymbol{u}^{\infty}\right|_{n_{m}}\right)\right]
\end{array}\right]
$$

where (24) follows by the definition of the subsequence $n_{m}$, the rest of the steps follow the same argument as in Section II-B, and $\Delta^{n_{m}}$ is defined in (8).

Following the same argument as above, we can modify the calculation in Section II-B to show that

$$
J^{\infty}\left(\tilde{\gamma}_{i}, \gamma_{-i}^{\infty}\right)-J^{\infty}\left(\gamma^{\infty}\right) \geq \limsup _{m \rightarrow \infty} \Delta_{i}^{n_{m}}\left(\left.\gamma^{\infty}\right|_{n_{m}},\left.\tilde{\gamma}^{\infty}\right|_{n_{m}}\right)
$$

Remark 5 Unlike the finite player setup, the sufficient condition for person-by-person optimality of a policy $\gamma^{\infty}$ :

$$
\lim _{m \rightarrow \infty} \Delta_{i}^{n_{m}}\left(\left.\boldsymbol{\gamma}^{\infty}\right|_{n_{m}},\left.\tilde{\gamma}^{\infty}\right|_{n_{m}}\right) \geq 0, \quad \forall i
$$

does not imply the sufficient condition for global optimality:

$$
\lim _{m \rightarrow \infty} \Delta^{n_{m}}\left(\left.\gamma^{\infty}\right|_{n_{m}},\left.\tilde{\gamma}^{\infty}\right|_{n_{m}}\right) \geq 0 .
$$

An alternative sufficient condition for global optimality is as follows.

Theorem 1 Let $\left\{\gamma^{n}\right\}_{n=0}^{\infty}$ be a sequence of strategies such that for all $\tilde{\gamma}^{n}$ and for all $i, \Delta_{i}^{n}\left(\gamma^{n}, \tilde{\gamma}^{n}\right) \geq 0$. Assume that:

(A1) there exists a strategy $\gamma^{\infty}$ such that

$$
\begin{aligned}
\limsup _{n \rightarrow \infty} \frac{1}{n} \mathbb{E}\left[c^{n}\left(\left.\boldsymbol{w}^{\infty}\right|_{n}, \boldsymbol{\gamma}^{n}\right)\right] \\
\quad=\limsup _{n \rightarrow \infty} \frac{1}{n} \mathbb{E}\left[c^{n}\left(\left.\boldsymbol{w}^{\infty}\right|_{n},\left.\boldsymbol{\gamma}^{\infty}\right|_{n}\right)\right] .
\end{aligned}
$$

Then under (A1), the strategy $\gamma^{\infty}$ is globally optimal for the infinite horizon setup.

Proof Let $\Gamma^{n}$ and $\Gamma^{\infty}$ denote the set of all strategies for the $n$-player and the infinite player teams, respectively. By Remark 3, we know that $\gamma^{n}$ is globally optimal for the $n$ player setup.

Assume that (A1) is true. Consider

$$
\begin{aligned}
J^{\infty}\left(\boldsymbol{\gamma}^{\infty}\right) & =\limsup _{n \rightarrow \infty} \frac{1}{n} \mathbb{E}\left[c^{n}\left(\left.\boldsymbol{w}^{\infty}\right|_{n},\left.\boldsymbol{\gamma}^{\infty}\right|_{n}\right)\right] \\
& =\limsup _{n \rightarrow \infty} \frac{1}{n} \mathbb{E}\left[c^{n}\left(\left.\boldsymbol{w}^{\infty}\right|_{n}, \boldsymbol{\gamma}^{n}\right)\right] \\
& =\limsup _{n \rightarrow \infty} \inf _{\tilde{\boldsymbol{\gamma}}^{n} \in \Gamma^{n}} \frac{1}{n} \mathbb{E}\left[c^{n}\left(\left.\boldsymbol{w}^{\infty}\right|_{n}, \tilde{\boldsymbol{\gamma}}^{n}\right)\right] \\
& =\limsup _{n \rightarrow \infty} \inf _{\tilde{\boldsymbol{\gamma}}^{\infty} \in \Gamma^{\infty}} \frac{1}{n} \mathbb{E}\left[c^{n}\left(\left.\boldsymbol{w}^{\infty}\right|_{n},\left.\tilde{\boldsymbol{\gamma}}^{\infty}\right|_{n}\right)\right] \\
& \leq \inf _{\tilde{\boldsymbol{\gamma}}^{\infty} \in \Gamma^{\infty}} \limsup _{n \rightarrow \infty} \frac{1}{n} \mathbb{E}\left[c^{n}\left(\left.\boldsymbol{w}^{\infty}\right|_{n},\left.\tilde{\boldsymbol{\gamma}}^{\infty}\right|_{n}\right)\right] \\
& =\inf _{\tilde{\boldsymbol{\gamma}}^{\infty} \in \Gamma^{\infty}} J^{\infty}\left(\tilde{\boldsymbol{\gamma}}^{\infty}\right)
\end{aligned}
$$

where (27) follows from (A1), (28) follows because $\gamma^{n}$ is globally optimal for team with $n$ players, (29) follows because $\left\{\left.\tilde{\gamma}^{\infty}\right|_{n}: \tilde{\gamma}^{\infty} \in \Gamma^{\infty}\right\}=\Gamma^{n}$, and (30) implies that $\gamma^{\infty}$ is optimal.

\section{CHARACTERIZATION OF PERSON BY PERSON LINEAR OPTIMAL POLICIES}

In this section we describe a class of parameters for which (A1) holds and we also outline a method to obtain $\gamma^{\infty}$.

Definition 3 Two sequences of vectors $\left\{v^{n}\right\}_{n=1}^{\infty}$ and $\left\{z^{n}\right\}_{n=1}^{\infty}$, where the terms $v^{n}$ and $z^{n}$ have the same dimension, are said to be asymptotically equivalent if $\lim _{n \rightarrow \infty} \frac{1}{\sqrt{n}}\left\|v^{n}-z^{n}\right\|_{2}=0$.

Definition 4 A sequence of vectors $\left\{v^{\wp, n}\right\}_{n=1}^{\infty}$ is periodic if there exists $\tilde{v}$ so that $v^{\wp, n}=\left[\tilde{v}^{T} \cdots \tilde{v}^{T}\right]^{T}$ whenever the dimension of $v^{\wp, n}$ is a multiple of the dimension of $\tilde{v}$. Here, we use $\wp$ to indicate that the sequence is periodic.

Assumption (A2) Assume the following:

1) There exists a sequence of periodic vectors $\left\{\eta^{\wp, n}\right\}_{n=1}^{\infty}$ satisfying $\eta^{\wp, n+m}=\left[\begin{array}{ll}\left.\eta^{\wp, n}\right)^{T} & \tilde{\eta}^{T}\end{array}\right]^{T}$, such that $\left\{\eta^{\wp, n}\right\}_{n=1}^{\infty}$ is asymptotically equivalent to $\eta^{n}$ as defined in (17).

2) There exist positive constants $\underline{\beta}$ and $\bar{\beta}$ so that $\left\|\left(\Gamma^{n}\right)^{-1}\right\| \leq \underline{\beta}$ and $\left\|\Gamma^{n}\right\| \leq \bar{\beta}$ holds for every $n$, where the matrix norm for $L \in \mathbb{R}^{m \times m}$ is defined as:

$$
\|L\|=\sup _{\left\{x \in \mathbb{R}^{m} \mid x^{\top} x=1\right\}}\left(x^{\top} L^{\top} L x\right)^{1 / 2} .
$$

3) There exists an integer $q$ and a square matrix $F$ such that the sequence $\left\{\eta^{\wp, m q}\right\}_{m=1}^{\infty}$ is asymptotically equivalent to the sequence $\left\{\Gamma^{m q}\left(I^{m} \otimes F\right) \eta^{\wp, m q}\right\}_{m=1}^{\infty}$, where $I^{m} \otimes F$ has the same dimension of $\Gamma^{m q}$ for every $m$. 
Theorem 2 If (A2) holds then (A1) holds. A corresponding $\gamma^{\infty}$ is given by $\gamma_{i}^{\infty}: y_{i} \mapsto H_{i}^{\wp, m q} y_{i}$, with $m q \geq i$, and $H_{i}^{\wp, m q}$ computed from

$$
\vec{H}^{\wp, m q}=\left(I^{m} \otimes F\right) \eta^{\wp, m q}, m \geq 1
$$

where $\vec{H}^{\wp, m q}$ is also a periodic vector defined as

$$
\vec{H}^{\wp, m q}=\operatorname{vec}\left[\left(H_{q}^{\wp, m q}\right)^{T}|\cdots| H_{m q}^{\wp, m q}\right] .
$$

Proof Our first goal is to show that the following holds:

$$
\lim _{m \rightarrow \infty} \frac{1}{\sqrt{m}}\left\|\vec{H}^{m q}-\vec{H}^{\wp, m q}\right\|_{2}=0
$$

Use the triangle inequality to write:

$$
\begin{aligned}
&\left\|\vec{H}^{m q}-\vec{H}^{\wp, m q}\right\|_{2}=\left\|\left(\Gamma^{m q}\right)^{-1} \eta^{m q}-\left(I^{m} \otimes F\right) \eta^{\wp, m q}\right\|_{2} \\
& \leq\left\|\left(\Gamma^{m q}\right)^{-1}\right\|\left\|\eta^{m q}-\eta^{\wp, m q}\right\|_{2} \\
&+\left\|\left(\Gamma^{m q}\right)^{-1}\right\|\left\|\eta^{\wp, m q}-\Gamma^{m q}\left(I^{m} \otimes F\right) \eta^{\wp, m q}\right\|_{2}
\end{aligned}
$$

From (33) and (34), we conclude the following:

$$
\begin{aligned}
\frac{1}{\sqrt{m}}\left\|\vec{H}^{m q}-\vec{H}^{\wp, m q}\right\|_{2} \leq \frac{\underline{\beta}}{\sqrt{m}}\left(\left\|\eta^{m q}-\eta^{\wp, m q}\right\|_{2}\right. \\
\left.+\left\|\eta^{\wp, m q}-\Gamma^{m q}\left(I^{m} \otimes F\right) \eta^{\wp, m q}\right\|_{2}\right)
\end{aligned}
$$

whose right hand side converges to zero due to (A2). It remains now to prove that (33) implies (A1). This will be achieved in two steps, where we prove that the following two limits hold:

$$
\begin{gathered}
\lim _{m \rightarrow \infty} \frac{1}{m q}\left[\left(\vec{H}^{m q}\right)^{\top} \Gamma^{m q} \vec{H}^{m q}-\left(\vec{H}^{\wp, m q}\right)^{\top} \Gamma^{m q} \vec{H}^{\wp, m q}\right]=0 \\
\lim _{m \rightarrow \infty} \frac{1}{m q}\left[\left(\vec{H}^{m q}-\vec{H}^{\wp, m q}\right)^{\top} \eta^{\wp, m q}\right]=0
\end{gathered}
$$

Notice that (35) and (36) imply (A1) because they show that both the quadratic and linear terms of the cost in (18) converge.

In order to show (35), we use the triangle inequality to write:

$$
\begin{gathered}
\frac{1}{\sqrt{m q}}\left|\left(\left(\vec{H}^{m q}\right)^{\top} \Gamma^{m q} \vec{H}^{m q}\right)^{1 / 2}-\left(\left(\vec{H}^{\wp, m q}\right)^{\top} \Gamma^{m q} \vec{H}^{\wp, m q}\right)^{1 / 2}\right| \\
\leq \frac{\bar{\beta}}{\sqrt{m q}}\left\|\vec{H}^{m q}-\vec{H}^{\wp, m q}\right\|_{2}
\end{gathered}
$$

which, from (33), converges to zero as $m$ tends to infinity.

Now, apply Hölder's inequality to the numerator of (36):

$$
\left|\left(\vec{H}^{m q}-\vec{H}^{\wp, m q}\right)^{\top} \eta^{\wp, m q}\right| \leq\left\|\vec{H}^{m q}-\vec{H}^{\wp, m q}\right\|_{1}\left\|\eta^{\wp, m q}\right\|_{\infty} .
$$

In order to show (36), notice that $\left\|\eta^{\wp, m q}\right\|_{\infty}$ in (37) is bounded because the vector is periodic and that the following holds:

$$
\begin{aligned}
\lim _{m \rightarrow \infty} \frac{1}{m q}\left\|\vec{H}^{m q}-\vec{H}^{\wp, m q}\right\|_{2}^{2}=0 \\
\Longrightarrow \lim _{m \rightarrow \infty} \frac{1}{m q}\left\|\vec{H}^{m q}-\vec{H}^{\wp, m q}\right\|_{1}=0
\end{aligned}
$$

where the left limit corresponds to (33).

\section{A. The Intuition Behind Assumption (A2)}

As the example in Section IV illustrates, when (1)-(2) correspond to the static reduction of the decentralized control of $q$ LTI subsystems then $\eta^{n}$ is asymptotically equivalent to a periodic vector. In our example, the process and measurement noise vectors affecting the overall system are stationary, and the boundary effect created by the initial condition causes the transient deviation that $\eta^{n}$ has from its periodic equivalent. An alternative characterization in terms of correlation matrices is also possible, where it can be shown that they will be asymptotically equivalent to block circulant matrices, as described in [19], [20]. A similar justification can be given to argue that $\Gamma^{n}$ and $\left(\Gamma^{n}\right)^{-1}$ are both asymptotically equivalent to block circulant matrices in the sense defined in [19], [20]. Hence, if the asymptotic periodicities of $\eta^{m q}$ and $\left(\Gamma^{m q}\right)^{-1}$ are compatible then the product $\left(\Gamma^{m q}\right)^{-1} \eta^{m q}$ will be asymptotically equivalent to the periodic vector given by $\left(I^{m} \otimes F\right) \eta^{\wp, m q}$ for some matrix $F$. In the particular case when $F$ is a scalar, this is the asymptotic equivalent of the fact that any constant vector is an eigenvector of a circulant matrix.

\section{EXAMPLE}

To illustrate the theory, we consider an example in which we first describe a $n$-player static team and then consider the optimal strategy as $n \rightarrow \infty$.

\section{A. Overall structure}

Let $\boldsymbol{w}=\left(w_{1}, w_{2}, \ldots\right)$ be a zero-mean wide-sense stationary Gaussian process with correlation matrix $\Sigma$. Consider the following $n$-player team: $w_{0}^{n}=0$ and for $i=1, \ldots, n$, $w_{i}^{n}=w_{i}$, the observations $y_{i}^{n}=w_{i}^{n}$, and hence the controls $u_{i}^{n}=\gamma_{i}^{n}\left(y_{i}^{n}\right)=\gamma_{i}^{n}\left(w_{i}\right)$. Thus, the correlation matrices $R^{n}$ and $S^{n}$ defined in Proposition 1 are given by $R^{n}=S^{n}=$ $\Sigma^{n}$, where $\Sigma^{n}$ is the upper $n \times n$ block of $\Sigma$.

Let $x_{i}^{n}=u_{i}^{n}+w_{i}^{n}$ and consider the cost function

$$
c\left(\boldsymbol{w}^{n}, \boldsymbol{u}^{n}\right)=\sum_{i=1}^{n} \sum_{j=1}^{n} x_{i}^{n} \hat{Q}_{i j}^{n} x_{j}^{n}+\sum_{i=1}^{n} \sum_{k=1}^{n} x_{i}^{n} \hat{P}_{i k}^{n} u_{k}^{n} .
$$

The above system is completely characterized by the matrices $\Sigma, \hat{Q}^{n}$, and $\hat{P}^{n}$. One can consider a series of examples with specific choices of these matrices. Due to lack of space, we consider only one example here

\section{B. Example (Coupled neighbors)}

As an example, consider the case when the cost of a player is coupled with that of its neighbors. In particular, let $\hat{Q}^{n}=$ $q \mathbb{I}^{n}$,

$$
\hat{P}^{n}=\left[\begin{array}{ccccc}
p & \hat{p} & 0 & \ldots & 0 \\
\hat{p} & p & \hat{p} & \ldots & 0 \\
0 & \hat{p} & p & \ldots & 0 \\
\vdots & \ddots & \ddots & \ddots & \vdots \\
0 & 0 & \ldots & \hat{p} & p
\end{array}\right],
$$

and $\Sigma_{i i}=\sigma^{2}, \Sigma_{i(i+1)}=\beta \sigma^{2}$. In this example, the result does not depend on the value of $\Sigma_{i j},|i-j|>1$, so we 
leave them unspecified. Further assume that $\exists \varepsilon$ such that $[|q+p|-2|\hat{p} \beta|] \sigma^{2}>\varepsilon$.

By substituting the value of $\hat{Q}^{n}$ and $\hat{P}^{n}$ in (39) and simplifying, we get that $Q^{n}$ and $P^{n}$ are tri-banded Toeplitz matrices in which for $i=1, \ldots, n$, and $j \in \sigma(i)$

$$
\begin{aligned}
Q_{i j}^{n} & =q+p, & Q_{i j}^{n} & =\hat{p} \\
P_{i j}^{n} & =2 q+p, & P_{i j}^{n} & =\hat{p}
\end{aligned}
$$

where $\sigma(1)=\{2\}, \sigma(n)=\{n-1\}$, and for $i=1, \ldots, n$, $\sigma(i)=\{i-1, i+1\}$.

Using the definitions in Corollary (1), we get that $\Gamma^{n}$ and $\eta^{n}$ are given as follows. For $i=1, \ldots, n$ and $j \in \sigma(i)$,

$$
\Gamma_{i i}^{n}=(q+p) \sigma^{2} \quad \text { and } \Gamma_{i j}^{n}=\hat{p} \beta \sigma^{2} .
$$

Moreover, $\eta_{1}^{n}=\eta_{n}^{n}=[2 q+p+\hat{p} \beta] \sigma^{2}$, and for $i=2, \ldots$, $n-1, \eta_{i}^{n}=[2 q+p+2 \hat{p} \beta] \sigma^{2}$,

Define

$$
\eta^{\wp, m}=[2 q+p+2 \hat{p} \beta] \sigma^{2} \mathbb{1}^{m} .
$$

$\eta^{\wp, n}$ is a periodic vector that differs from $\eta^{n}$ only at the first and the last components. Therefore, $\eta^{\wp, n}$ is asymptotically equally distributed as $\eta^{n}$ and, hence, (A2-1) is satisfied.

$\Gamma^{n}$ is a tri-banded Toeplitz matrix. Therefore, (A2-2) is satisfied if (see [21]): $\exists \varepsilon$ such that $\left[|q+p|-2|\hat{p} \beta| \mid \sigma^{2}>\varepsilon\right.$. We assume that such an $\varepsilon$ exists, and therefore (A2-2) is satisfied.

Define $F=\left[F_{11}\right]$ to be a $1 \times 1$ matrix such that

$$
F_{11}=\frac{1}{[q+p+2 \hat{p} \beta] \sigma^{2}}
$$

Then, for any $m \geq 1$

$$
\left(\mathbb{I}^{m} \otimes F\right) \eta^{\wp, m}=\frac{2 q+p+2 \hat{p} \beta}{q+p+2 \hat{p} \beta} \mathbb{1}^{m} .
$$

For ease of notation, define $\mu^{m}=\Gamma^{m}\left(\mathbb{I}^{m} \otimes F\right) \eta^{\wp, m}$. Then,

$\mu_{1}^{m}=\mu_{m}^{m}=(q+p+\hat{p} \beta)(2 q+p+2 \hat{p} \beta) \sigma^{2} /(q+p+2 \hat{p} \beta)$

and for $i=2, \ldots, m-1$

$$
\mu_{i}^{n}=(2 q+p+2 \hat{p} \beta) \sigma^{2} .
$$

Note that $\mu^{n}$ differs from $\eta^{\wp, m}$ only at the first and the last components. Therefore, $\mu^{m}$ is asymptotically equally distributed as $\eta^{\wp, m}$ and, hence, (A2-3) is satisfied.

Thus, $\eta^{\wp, m}$ and $F$ defined above satisfy (A2). Therefore, by Theorem 2 ,

$$
\vec{H}_{i}^{\wp, m}=\frac{2 q+p+2 \hat{p} \beta}{q+p+2 \hat{p} \beta}
$$

and, consequently, an optimal strategy is given by

$$
\begin{gathered}
\gamma_{i}^{\infty}: w_{i} \mapsto \frac{2 q+p+2 \hat{p} \beta}{q+p+2 \hat{p} \beta} w_{i} . \\
\text { V. CONCLUSION }
\end{gathered}
$$

\section{CONCLUSION}

In this paper we established sufficient conditions for the optimality of person-by-person-optimal policies in static teams with countably infinite players. The application of these results to infinite horizon, partially nested stochastic dynamic teams is currently under investigation.

\section{REFERENCES}

[1] H. S. Witsenhausen, "The intrinsic model for discrete stochastic control: Some open problems," in Control Theory, Numerical Methods and Computer System Modelling. A. Bensoussan and J. L. Lions Springer Verlag 107: Lecture Notes in Economics and Mathematical Systems, 1975, pp. 322-335.

[2] J. Marschak, "Elements for a theory of teams," Management Science, vol. 1, pp. 127-137, 1955.

[3] R. Radner, "Team decision problems," Ann. Math. Statist., vol. 33, pp. $857-881,1962$

[4] J. Marshak and R. Radner, Economic Theory of Teams. New Haven, CT: Yale University Press, 1972.

[5] H. Witsenhausen, "Separation of estimation and control for discrete time systems," Proceedings of the IEEE, vol. 59, pp. 1557-1566, 1971.

[6] _ _ "Equivalent stochastic control problems," Math. Control, Signals and Systems, vol. 1, pp. 3-11, 1988.

[7] H. S. Witsenhausen, "A standard form for sequential stochastic control," Mathematical Systems Theory, vol. 7, pp. 5-11, 1973.

[8] - "On information structures, feedback and causality," SIAM J. Control, vol. 9, pp. 149-160, May 1971.

[9] S. Yüksel and T. Başar, Stochastic Networked Control Systems: Stabilization and Optimization under Information Constraints. Boston, MA: Birkhäuser, 2013

[10] J. Krainak, J. Speyer, and S. Marcus, "Static team problems - part II Affine control laws, projections, algorithms, and the LEGT problem,' IEEE Transactions Automatic Contr., vol. 27, pp. 848-859, 1982

[11] Y. C. Ho and K. C. Chu, "Team decision theory and information structures in optimal control problems - part I," IEEE Transactions on Automatic Control, vol. 17, pp. 15-22, February 1972.

[12] A. Mahajan, N. Martins, M. Rotkowitz, and S. Yüksel, "Information structures in optimal decentralized control," in IEEE Conference on Decision and Control, Hawaii, USA, 2012.

[13] B. Bamieh and P. Voulgaris, "A convex characterization of distributed control problems in spatially invariant systems with communication constraints," Systems Cont. Letters, vol. 54, pp. 575-583, 2005.

[14] M. Rotkowitz and S. Lall, "A characterization of convex problems in decentralized control," IEEE Transactions on Automatic Control, vol. 51, pp. 274-286, February 2006.

[15] L. Lessard and S. Lall, "A state-space solution to the two-player decentralized optimal control problem." Monticello, IL: Annual Allerton Conference on Communication, Control and Computing, 2011

[16] S. Sabau and N. C. Martins, "Stabilizability and norm-optimal control design subject to sparsity constraints," arXiv.

[17] F. Lin, M. Fardad, , and M. R. Jovanovic, "Optimal control of vehicular formations with nearest neighbor interactions," IEEE Transactions on Automatic Control, vol. 57, pp. 2203-2218, September 2012.

[18] N. Sandell and M. Athans, "Solution of some nonclassical LQG stochastic decision problems," IEEE Transactions on Automatic Control, pp. 108-116, 1974.

[19] R. M. Gray, Toeplitz and Circulant Matrices: A review (Foundations and Trends in Communications and Information The). Now Publishers Inc, 2006

[20] U. Grenander and G. Szego, Toeplitz Forms and Their Applications (AMS Chelsea Publishing). American Mathematical Society, 2001.

[21] A. Bottcher and S. Grudsky, Spectral Properties of Banded Toeplitz Matrices. SIAM, 2005. 